\title{
Introduction and preliminary results of a calibration for full-frame hyperspectral cameras to monitor agricultural crops with UAVs
}

\author{
H. Aasen ${ }^{\text {a, }}$, J. Bendig ${ }^{\text {a }}$, A. Bolten ${ }^{\text {a }}$, S. Bennertz ${ }^{\text {a }}$, M. Willkomm ${ }^{\text {a }}$ and G. Bareth ${ }^{\text {a }}$ \\ ${ }^{a}$ Department of Geoscienece, University of Colgone, Germany - (helge.aasen, juliane.bendig, andreas.bolten, m.willkomm, \\ g.bareth)@uni-koeln.de, s.bennertz@gmail.com
}

KEY WORDS: hyperspectral, full-frame imager, UAV, vegetation, agricultural crops, precision agriculture

\begin{abstract}
:
Hyperspectral remote sensing helps to acquire information about the status of agricultural crops to allow optimized management practices in the context of precision agriculture. Due to technological innovations small and lightweight hyperspectral sensors have become available which may be carried by unmanned aerial vehicles (UAVs). In this paper we give a brief overview over existing hyperspectral sensors for UAVs. We focus on a new type of full-frame sensors which capture hyperspectral information in two dimensional image frames. We then develop a calibration procedure for these sensors and identify challenges in remote sensing of vegetation. The calibration is evaluate by in-field data acquired during a flight campaign. The spectral calibration shows good results with less than three percent difference in reflection for 110 of the 125 bands (458 to $886 \mathrm{~nm}$ ).
\end{abstract}

\section{INTRODUCTION}

In consideration of the increasing human population, sharpened by extended meat and dairy consumption and biofuel production, Ray et al. (2013) concluded, that the agricultural production needs to be roughly doubled by 2050 or increased by about 2.4 percent per year. At the same time expending agricultural areas have far-reaching effects on the ecosystem (Foley et al., 2005). Thus the agricultural production needs to intensify to increase crop production while reducing unsustainable uses of water, nutrients and agricultural chemicals (Foley et al., 2011).

Precision agriculture aims at more efficient use of resources by optimizing the management practice of agricultural crops to apply treatments at the right amount, place and time (Mulla, 2013). To archive this, in-time knowledge of the crops status is needed (Atzberger, 2013).

Hyperspectral data has shown great potential to derive information about the biophysical (Aasen et al., 2014; Erdle et al., 2011; Gnyp et al., 2013; Hansen and Schjoerring, 2003) and biochemical parameters ( $\mathrm{Li}$ et al., 2010; Yu et al., 2013b) of agricultural crops as well as to detect environmental stress or plant diseases (Delalieux et al., 2007; Mahlein et al., 2013; Stagakis et al., 2012; Yu et al., 2013a). Traditionally, hyperspectral data is acquired with field spectrometers, airborne sensors or satellites (Green and Eastwood, 1998; Milton et al., 2009; Pearlman et al., 2003).

Unmanned aerial vehicles (UAVs) represent a flexible carrier platform for different sensors. They are easily transported to an area of interest and used for in-time data acquisition. In comparison with satellite and high flying airborne platforms UAVs fly below the clouds. Thus, optical remote sensing with UAVs is not limited to clear sky conditions - provided robust calibration methods to account for changing illumination with changing cloud cover. Fixed wing (airplane-like) UAVs can cover large areas while multi-rotor (helicopter-like) UAVs have no minimum speed and allow complex flight patterns and stationary measurements.

With technological innovations, hyperspectral sensors have been shrinking in size and weight and a have thus become feasible for use onboard of UAVs. Burkart et al. (2014) introduced an ultralight weight spectrometer mounted on a UAV for field spectroscopy. Several line scanners are available for UAVs and have been flown on fixed-wing (Berni et al., 2009; Hruska et al., 2012) and multi-rotor UAV (Lucieer et al., 2014) for vegetation studies.

Recently, a new type of hyperspectral sensors was introduced that records a full hyperspectral image frame (full-frame). In contrast to line scanners, which create line-frames with one spatial and one spectral dimension, full-frame hyperspectral sensors record a full image with two spatial dimensions and one spectral dimension. Currently, two systems are available for UAVs. The Rikola (http://www.rikola.fi/) Fabry-Perot interferometer (FPI) uses an adjustable air gap to record selectable spectral bands in the wavelength range from 400 to $1000 \mathrm{~nm}$ with a spatial resolution (in default binning) of 1024 by 648 pixels (Honkavaara et al., 2013).

The second available full-frame hyperspectral imager is the Cubert UHD 185 - Firefly (http://cubert-gmbh.de/). This camera simultaneously records 125 bands between 450 and $950 \mathrm{~nm}$ with 50 by 50 hyperspectral pixels within one frame acquisition. At the same time a grayscale image with about one megapixel is recorded. With the software provided by the manufacturer the hyperspectral pixel may be pan-sharpened to the grayscale image's resolution. The latter sensor is used in this study and further details are given in section 2.1. A third light weight hyperspectral full-frame camera has been presented by BaySpec (http://www.bayspec.com/). To the authors knowledge this sensor has not been used with an UAV so far.

Due to the weight and size limitation for hyperspectral sensor systems carried by a UAV, several challenges exist for vegetation and agricultural crop monitoring:

- Since the sensor is mounted on a moving platform the image acquisition has to be fast enough to avoid image blur and spectrally mixed pixels

- $\quad$ Since the light reflected by plants in the near infrared (NIR) is many magnitudes stronger than in the visible (VIS) the signal to noise ratio and the dynamic range of the sensor have to be high enough to capture the reflection variances in the in both regions.

- Since slight changes in the reflectance of plants may point out changes in their biophysical and biochemical parameters, the sensors need to be spectrally well calibrated. 
Due to the fact that full-frame hyperspectral sensors (spatially) record two dimensional images, orthorectification and georeferencing is simpler than with line scanners. However, this comes with the disadvantage that during the sensor calibration process, besides of the spectral dimension, two spatial dimensions have to be taken into account instead of one. At the same time the complex optical system of such sensors introduce an additional source of error to the whole system. Thus, the data recorded has to be carefully examined and interferences have to be corrected with appropriate calibration procedures. This procedure is critical to provide high quality data products that can be used for vegetation studies. The calibration procedure becomes even more important if results from different sensors (e. g. field spectrometers, UAV, airborne and satellite sensors) should be comparable among each other.

During image acquisition the reflectance of an object is influenced by a combination of environmental effects (EE), sensor effects (SE) and the objects properties (OP). For vegetation and agricultural crops, the $\mathrm{OP}$ are the biochemical or biophysical properties of the plant.

EE include the condition of the atmosphere, bi-directional reflectance effects influenced by the sun-object-sensor arrangement including (micro-) topography, surface conditions (e.g. surface wetting) and, in case of passive sensors, illumination changes. SE are errors by the sensors charge-coupled device (CCD) detector and the electronically components and optical system.

In this paper we present a calibration method for a full-frame hyperspectral sensor for the measurement of the surface reflectance signatures. Thus, we focus on correcting the SE influencing the signatures. Moreover only those EE influencing the path of the light from the object to the sensor will be discussed in this paper while others (e.g. surface condition) are accepted as part of the objects spectral properties.

We first present a calibration approach for the new type of fullframe hyperspectral camera. After presenting the equipment used for the calibration we evaluate the calibration approach using field data recorded during a flight.

\section{EQUIPMENT}

In this study we used the Mikrokopter OktoXL to carry the UHD 185 hyperspectral full-frame imaging system together with a mini computer to (remotely) control the camera and store the data. The calibration process of the camera was carried out in the lab with a Labsphere integration sphere.

\subsection{Cubert UHD 185-Firefly}

The UHD 185-Firefly is a hyperspectral full-frame camera which simultaneously captures 138 spectral bands with a sampling interval of $4 \mathrm{~nm}$. From these bands the camera's manufacturer recommends the use of 125 bands between 450 and $905 \mathrm{~nm}$. The full-width half-mean (FWHM) of the bands is shown in Figure 1. The FWHM increases from about $4 \mathrm{~nm}$ at $450 \mathrm{~nm}$ to about $25 \mathrm{~nm}$ at $810 \mathrm{~nm}$ (Cubert $\mathrm{GmbH}$, personal correspondence, 2014). For each band a 50 by 50 pixel image with 12 bit (4096 DN) precision is created. At the same time a grayscale image with a resolution of 990 by 1000 pixel is captured.

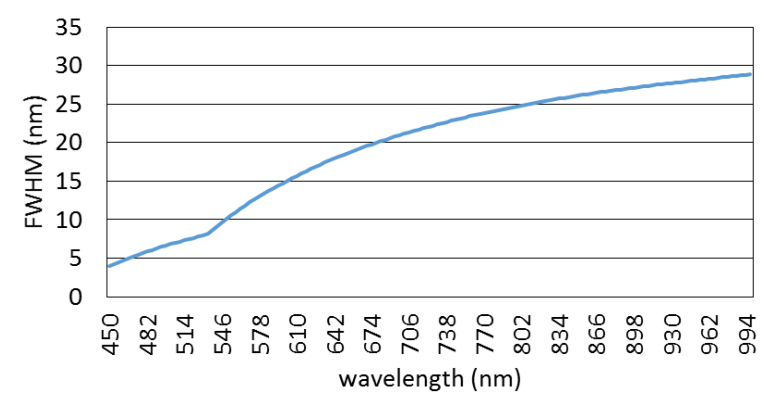

Figure 1. FWHM as given by the manufacturer

The camera housing is about 28 by 6.5 by $7 \mathrm{~cm}$. Inside a Si CCD is used as a detector. We use a lens with a focal length of $30 \mathrm{~mm}$ resulting in a field of view (FOV) of about $11^{\circ}$. Since the camera housing is elongated with the lens locking forward a mirror is fixed at the front to capture images in nadir orientation (Figure 2). The ground resolution at $30 \mathrm{~m}$ flying height is about $21 \mathrm{~cm}$ for the hyperspectral pixels and $1 \mathrm{~cm}$ for the grayscale image. With the software of the camera the hyperspectral resolution may be pan-sharpened to the grayscale images resolution. Latter was not used in our study. The total weight of the Camera is about 470 gr.

The camera is controlled by a single board computer (SBC). It is connected to the camera by two gigabit ethernet cables and runs a server application which takes care of the communication with the camera and the data storage. The configuration may be done remotely via WiFi from a control application run on a different computer. Within the WiFi range a live view may be transmitted to the control application and measurements may be triggered manually. Additionally, a sequence of images may be recorded with defined frequency and duration. In our case we used a Pokini $\mathrm{Z}$ (http://www.pokini.de/) as SBC. This combination allows to capture full-frame image cubes with a frame rate of about 0.6 hertz. The typical integration time under cloudless conditions is one millisecond (ms), increasing to about four ms under cloud covered conditions. The whole image capturing system (ICS) consisting of the camera, SBC and a separate lithium polymer battery weights about $1 \mathrm{~kg}$. The whole package is mounted on a gimbal carried by the UAV (Figure 2.)

\subsection{MikroKopter OktoXL}

The MK-OktoXL is a multi-rotor Mini-UAV (http://www.mikrokopter.de). The Mini-UAV carries a max. 2.5 $\mathrm{kg}$ payload, which results in a total weight of under $5 \mathrm{~kg}$. The payload consists of the sensor that is mounted on a gimbal. The gimbal compensates pitch and roll movement during the flight by using the UAV's on-board gyroscopes. The compensation allows to maintain a nadir orientation of the sensor. Depending on the payload and the batteries, the flight time varies from 15 to $30 \mathrm{~min}$. Flights are allowed within the line of sight, which equals several $100 \mathrm{~m}$. Altitude, speed and position are controlled during the flight and logged to an on-board memory card. The flight path is controlled by an autopilot that enables waypoint navigation. 


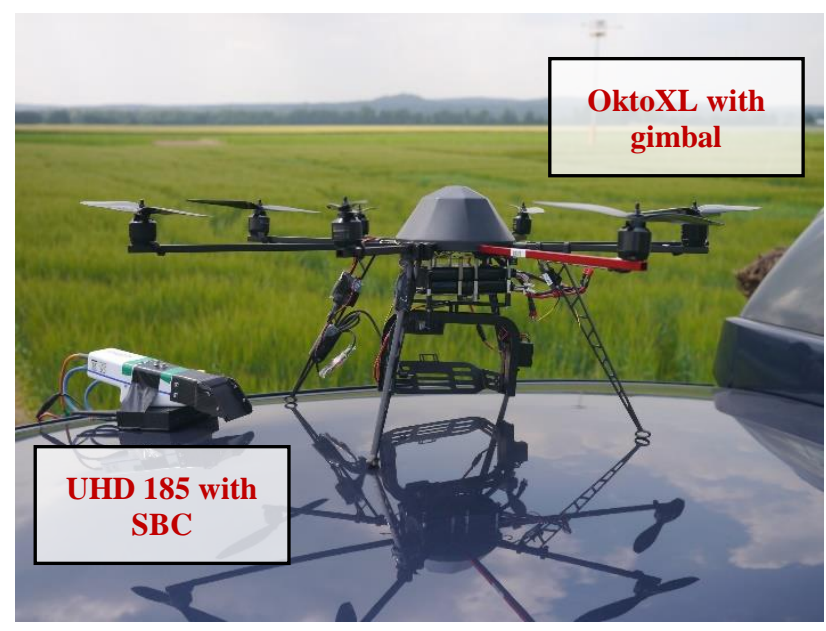

Figure 2: UHD 185 with SBC and OktoXL with gimbal

\subsection{Integration Sphere}

For the flat-field measurements an integration sphere of Labsphere (http://www.labsphere.com) was used (CSTM-USS1200C-SL). The sphere has four internal light sources: Three static 35 Watts and one dimmable 100 Watts. The integration sphere is calibrated such that it is homogenously illuminated when all lights are activated. The illumination is measured by a silicone and an InGaAS detector. Since we wanted to perform the test under typical in-field settings with about one ms integration time we only used two of the lights. More lights would have exceeded the dynamic range of the UHD 185 with this integration time. All measurements where performed in a dark room with all light turned off but the sphere.

\section{METHODS}

\subsection{Noise removal}

CCD sensors proportionally transform the incoming luminous energy of each point of a scene into an electrical signal (Mansouri et al., 2005). During the process of translating radiation into digital numbers (DN) noise is added to the signal. The noise becomes obvious when the camera is triggered under perfect dark conditions. Theoretically, the image DN values should be zero. Mostly, this is not the case due to the dark current (DC). The DC consists of a constant part (readout noise) and a non-constant part depending on the detectors temperature and the integration time (thermal noise). After measuring the DC (e.g. by obscuring the light entrance to the detector) it can be subtracted from the sensor's raw DN values. So far hyperspectral UAV sensors do not provide DC measurements in-flight. Due to its temperature dependency Burkart et al. (2014) developed a DC correction depending on the sensors temperature for his flying spectrometer. Unfortunately, the UHD 185 does not provide temperature logging so far. To approximate the magnitude of the thermal noise we captured 30 images under perfectly dark conditions at 13, 23 and 33 minutes after the sensor was switched on. We assumed an up-heating with time. We then calculated the average of the images for every image sequence. Because of the sensor design, different bands are measured at different parts of the CCD and may behave differently. To take this into account we estimated the noise by extracting the median values of the averaged images for four representative bands.

\subsection{Dynamic range and signal to noise ratio}

If the quantum of photons received by a CCD reaches a certain level the photodynamic cells start to saturate (the CCDs quantum efficiency decreases). As a result the transformation from incoming radiation to DN values is not linear anymore. For most detectors this level is below the theoretical dynamic range (e.g. 12 bit $=4096$ counts for the UHD 185). Careful extermination of the quantum efficiency has to be performed to avoid misinterpretation due to saturation effects. Consequently, the dynamic range should be limited to a level below the saturation (real dynamic range (rDR)). This limitation is particularly important for UAV remote sensing since illumination conditions may change quickly with cloud cover. Moreover, remote sensing of vegetation faces the challenge of low reflectance by plants in the VIS and high reflectance in the NIR. Thus, the rDR should be known and optimally used to use the biggest possible range of DN values (and improve the real SNR) for the VIS while avoiding saturation in the NIR.

To assess the saturation point of the UHD 185 we pointed the camera into the integration sphere and successively incremented the light intensity in 16 steps. At each step we took 30 images and later averaged these. From these images we extracted the DN values of 25 pixels at the center of the brightest band at $734 \mathrm{~nm}$. These values were averaged for each illumination step. To evaluate the linearity we divided the resulting DN values by the light intensity measured by the light detectors inside the sphere.

\subsection{Flat-fielding}

On the path through the optical system of the sensors the incoming radiance is altered by the vignetting effect. Vignetting is defined as a spatially dependent light intensity fall-off that results in a radial reduction in radiance towards the image edges (Goldman, 2010; Kim and Pollefeys, 2008). A more specific overview over different sources of vignetting can be found in Goldman (2010).

Vignetting may be corrected by modelling the optical pathway or by image-based techniques. We used the latter approach since it is both simpler and more accurate (Yu, 2004). It is based on the generation of per pixel coefficients stored into a look-up-table (LUT) to correct for illumination fall-off. This calibration is done by pointing the sensor at a perfect homogeneous, lambertian surface (flat field).

In our case we pointed the sensor inside the integration sphere. 30 images were taken with two lamps activated (one at 70 percent closure) to stay within the rDR. We averaged the images and used the median value of each band to generate the LUT for every pixel in the band. We chose the median to exclude extreme pixel values from the procedure and to minimize overcompensation by extreme coefficients. Besides the vignetting the optical system of the camera may influence the incoming radiation in other ways. As long as these are linear the LUT approach corrects for these influences as well.

\subsection{Transformation to reflectance}

After the SE correction the resulting DN values are transformed to reflectance values to ease further analysis. Additionally, the environmental conditions may change between different field campaigns, in-between flights and even within a flight. During the transformation these effects may be normalized making the data comparable.

For hyperspectral UAV remote sensing three main methods for $\mathrm{DN}$ to reflectance transformation are conceivable: 
a) Simultaneous irradiance measurements of the sun's radiation on the ground (Burkart et al. (2014)) or on the UAV,

b) In-flight measurements of spectrally well characterized targets on the ground (Lucieer et al. (2014)),

c) Pre-flight reference calibration with a spectrally well characterized target (Bareth et al., in review; Suomalainen et al., 2014).

For the pre-flight reference calibration the sensor is pointed at a spectrally homogenous target with a known reflectance lying on the ground and an image is taken. In the post-processing this reference image is used to transform the image's DN values captured during the flight to reflectance. Since the reference target is homogenous, the reference image should correct for the previously mentioned disturbances of the imagery by the optical system. However, careful assessment of the sensor-tocalibration-target geometry as well as stray light sources from objects and people surrounding the target are critical. Since the reference image will be used for the transformation of all images, errors during the measurement of the calibration target will influence all data. Additionally, no adaptation to changing light conditions during the flight is possible since another measurement may only be carried out again after the flight.

The pre-flight reference calibration with one calibration panel represents a one-point calibration. Burger and Geladi (2006) mentioned the beneficial effect of calibration targets with different grayscale intensities (multi-point calibration). Measuring multiple targets before the flight would add complexity to the pre-flight procedures and eventually reduce flight time due to battery consumption. In-flight measurements of calibration targets provide the opportunity to efficiently measure multiple targets under in-flight illumination conditions. However, these targets need to be big enough to cover several pixels. In the post-processing these targets are then identified, their spectra are extracted and conversion coefficients from DN to reflectance are calculated and applied to the imagery. To take into account changing illumination, multiple targets have to be spread out along the flight path or the targets have to be overflown several times during the flight.

More conveniently, continuous sun irradiance measurements may quantify the illumination change during the flight. If these measurements are ground based a spatial error may be introduced since the UAV might fly in an area with different conditions than what is measured on the ground. To minimize this error the irradiance sensor should be mounted on the UAV. The latter might be the best approach for the correction of illumination changes. However, the irradiance sensor would add additional weight and complexity to the sensor system.

For this study we modified the pre-flight reference calibration approach. Before the flight the sensor was pointed at a white Zenith Lite ${ }^{\mathrm{TM}}$ panel lying on the ground. The integration time was adapted to yield a maximum $\mathrm{DN}$ value of 3500 and a measurement was taken. We then applied a flat-fielding to the image of the Zenith Lite ${ }^{\mathrm{TM}}$. Since the sensor to panel geometry as well as the sun angle might introduce a bias we used the median of each band to minimize the influence of these effects. We multiplied the values by 1.042 since the Zenith Lite ${ }^{\mathrm{TM}}$ has a reflection coefficient of about 96 percent for the visible and NIR part of the spectrum. We used the resulting values as coefficients to transform the $\mathrm{DN}$ values to reflectance.

\section{RESULTS}

\subsection{Noise removal}

Figure 3 shows the DC for one ms integration time of four representative wavelength $(502,550,670,770 \mathrm{~nm})$ in relation to the time after the sensor was switched on. After $13 \mathrm{~min}$ the average dark current is about $0.41 \mathrm{DN}$. After 33 minutes the dark current increased by $0.55 \mathrm{DN}$ to an average value of $0.96 \mathrm{DN}$. The inter band difference is below $0.17 \mathrm{DN}$.

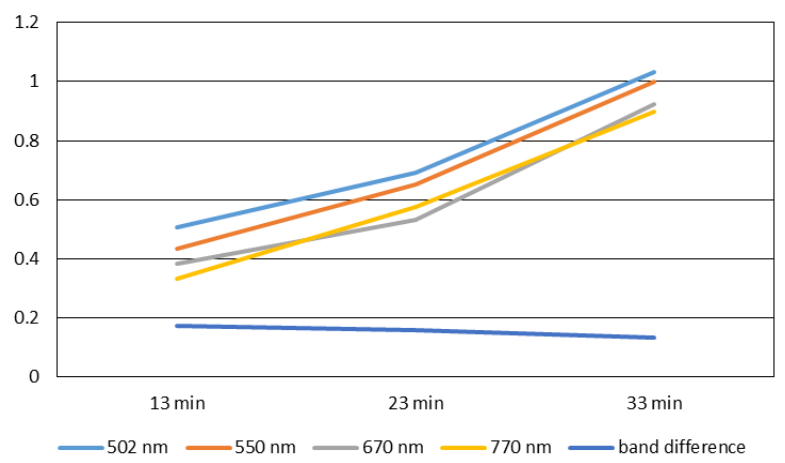

Figure 3. Dark current for $1 \mathrm{~ms}$ integration time in four different bands 13, 23, 33 min after sensor switch-on

Visual evaluation of the dark current measurements reveal slight spatial inhomogeneity of the DC within on band below one count. This pattern is also reflected in the standard deviation. After the sensor is heated up (33 $\mathrm{min})$ the standard deviation spatially equalize itself to a value of about 0.8 counts.

\subsection{Dynamic range and signal to noise ratio}

Figure 4 shows the measured DN values of the UHD 185 together with the ratio of the DN values divided by the light intensity measured inside the sphere. For the first two illumination steps the ratio is increasing. The third step corresponds to one illuminated lamp with a closure of 75 percent $\left(8.00 \mathrm{E}^{-5} \mathrm{~W} / \mathrm{m}^{2}\right)$. The ratio is quite stable until illumination step 12 . The measured DN at step 12 is about 3600 . After step 12 the ratio decreases. At step 13 the measured DN is about 3800 .

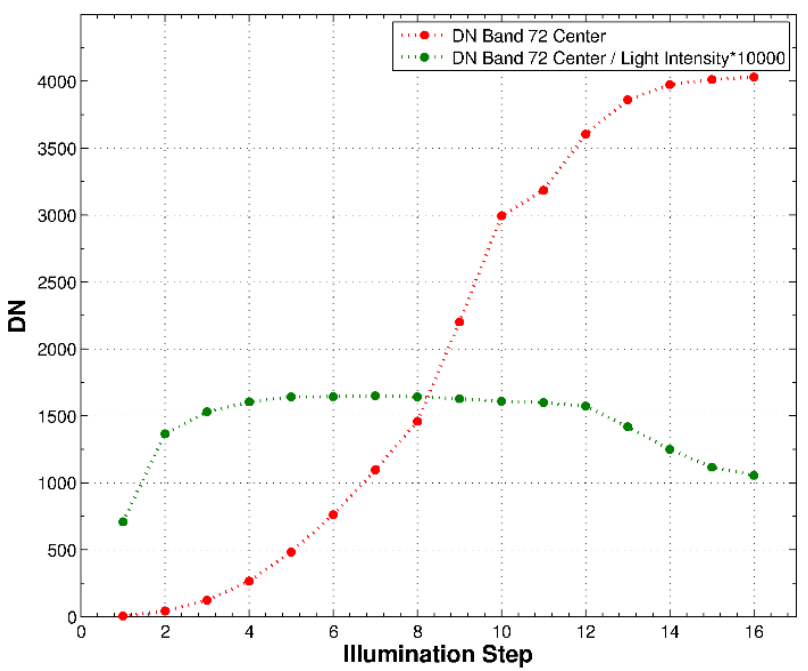

Figure 4: Measured DN (red) and ratio between DN and light intensity of the sphere (green). For a better visualization the latter value was multiplied by 10000

\subsection{Flat fielding}

Figure 5 shows the LUT coefficients for band $550 \mathrm{~nm}$ and 770 nm. A spherical fall-off of the DN values can be seen. Additionally, an undulated pattern is visible in the $\mathrm{x}$ direction. At $550 \mathrm{~nm}$ the DN values of the brightest and the darkest pixel differ by about 65 percent. At $770 \mathrm{~nm}$ they differ by about 90 percent. 


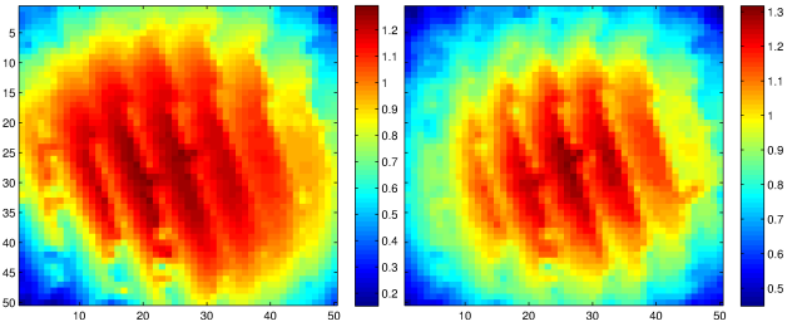

Figure 5. LUT coefficients at $550 \mathrm{~nm}$ (left) and $770 \mathrm{~nm}$ (right)

To validate the stability of the flat field calibration across different light intensities we applied the flat field coefficients stored in the LUT to measurements at different light intensities (Table 1). Overall, with doubling the light intensity the DN values have a range less than 8 percent relative to the mean with a coefficient of variation close to zero.

\begin{tabular}{|c|c|c|c|c|c|c|c|}
\hline $\begin{array}{c}\text { Light Int. } \\
\text { (\%) }\end{array}$ & $\min (\mathbf{D N})$ & $\max (\mathbf{D N})$ & range (DN) & mean (DN) & $\begin{array}{c}\text { range / } \\
\text { mean }\end{array}$ & std & cv \\
\hline 197.48 & 2464.10 & 2631.10 & 167.00 & 2541.20 & 0.07 & 25.08 & 0.01 \\
\hline 185.56 & 2332.90 & 2471.80 & 138.90 & 2398.30 & 0.06 & 21.66 & 0.01 \\
\hline 164.12 & 2074.20 & 2188.60 & 114.40 & 2129.50 & 0.05 & 16.73 & 0.01 \\
\hline 139.51 & 1787.90 & 1861.50 & 73.60 & 1822.80 & 0.04 & 10.37 & 0.01 \\
\hline 116.44 & 1522.50 & 1559.60 & 37.10 & 1539.30 & 0.02 & 4.78 & 0.00 \\
\hline 100.00 & 1341.20 & 1341.20 & 0.00 & 1341.20 & 0.00 & 0.00 & 0.00 \\
\hline 92.65 & 1239.70 & 1262.60 & 22.90 & 1252.20 & 0.02 & 3.16 & 0.00 \\
\hline 91.10 & 1223.10 & 1247.30 & 24.20 & 1235.70 & 0.02 & 3.28 & 0.00 \\
\hline
\end{tabular}

Table 1. Descriptive statistics of the LUT applied to different light intensities shown in percent of the calibration intensity (in gray).

Visual inspection reveals a spatial pattern that relatively increases with the change in light intensity compared to the calibration intensity (Figure 6, left). The difference of the brightest and darkest pixel is below 4 percent of the average DN. Additionally, strong gradients are observed in the top right corner with the highest and lowest $\mathrm{DN}$ values close to each other.

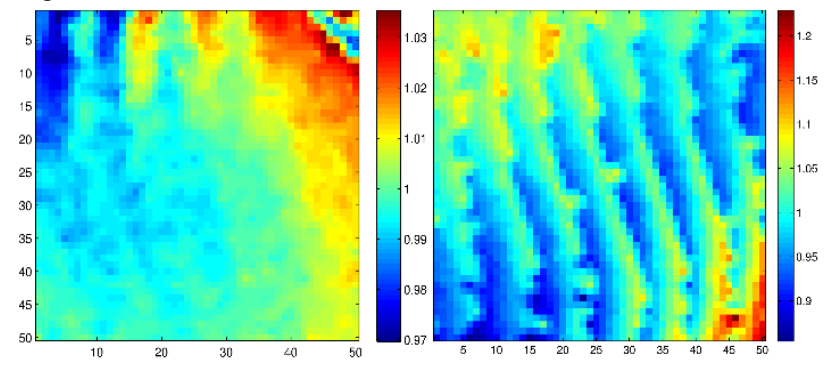

Figure 6. Relative DN offset with doubled light intensity compared to the calibration (left). Artefacts after applying the flat-field coefficients to an in-field measurement of a Zenith panel (right).

To further evaluate the flat-fielding we applied the flat-field coefficients to an image of a Zenith Lite ${ }^{\mathrm{TM}}$ panel (Figure 6, right). Visual inspection of the image reveal a residual undulated pattern in the $\mathrm{x}$ direction after the flat-fielding. Additionally, an underlying gradient with higher values across the image from the top left to the bottom right corner can be observed.

\subsection{Transformation to reflectance}

To evaluate the result of the modified pre-flight reference calibration we compared spectra of four 80 by $80 \mathrm{~cm}$ reference panels measured with an ASD FieldSpec 3 at $1 \mathrm{~m}$ above the ground and the UHD 185 flying at $30 \mathrm{~m}$ above ground. The integration time was set to $1 \mathrm{~ms}$. The experiment was carried out in early June near the city of Bonn, Germany, under blue sky conditions at about $1 \mathrm{pm}$ local time. The measurement of the preflight reference, the in-flight scene and the ASD took about 30 minutes.

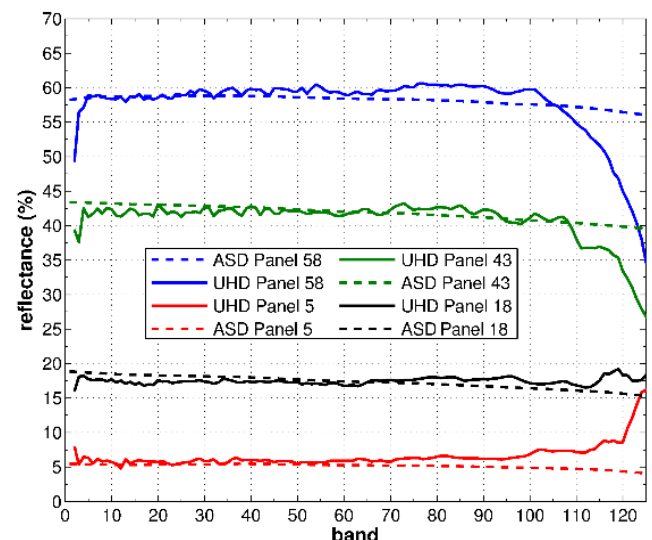

Figure 7. Comparison of the resampled ASD spectra (dashed) with the UHD spectra (solid) of band 1 to 125 for four spectral panels $(5,18,43$ and 58 percent reflectance).

We applied the modified pre-flight reference calibration to the scene captured by the UHD 185 . To compare the spectra we manually extracted the center pixel of each panel from the inflight scene. The ASD spectra were resampled using ENVI 5 (Exelis Visual Information Solutions, Boulder, Colorado) with the FWHM values of the UHD 185 provided by the manufacturer.

Bands 3 to 110 (458 to $886 \mathrm{~nm}$ ) show an absolute offset of less than 3 percent reflection, whereas most bands ( 3 to 75 ) show an offset of less than 2 percent (Figure 7). The average offset is less than 1 percent between band 1 and 110 . Overall, the UHD slightly underestimates the reflectance in the lower and slightly overestimates it in the higher bands.
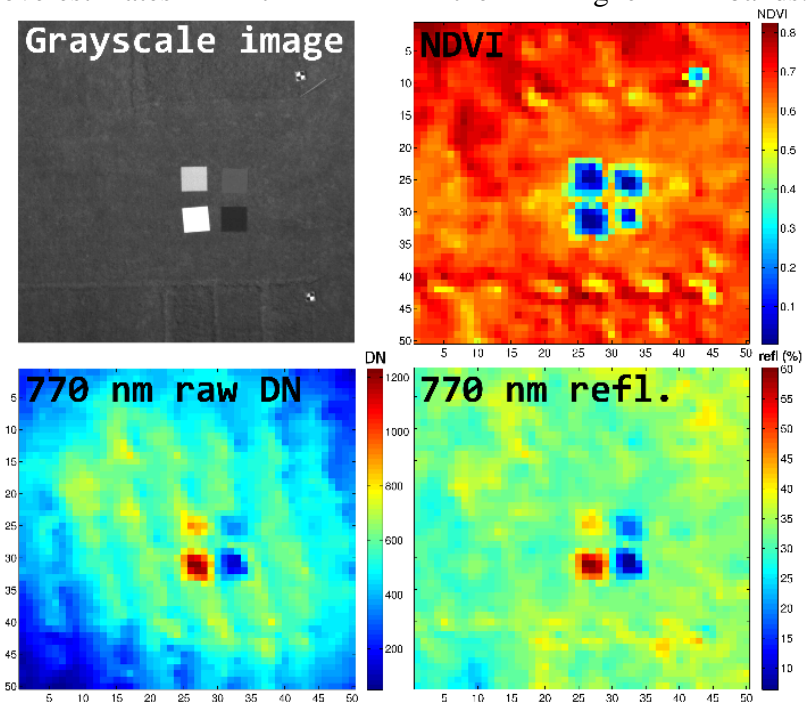

Figure 8. Grayscale image (top left), $770 \mathrm{~nm}$ raw DN and reflectance (bottom left and right) and NDVI from a scene with four calibration panels (top right).

Figure 8 shows the full scene for the $770 \mathrm{~nm}$ band before and after the calibration, a NDVI derived from the $670 \mathrm{~nm}$ and 770 $\mathrm{nm}$ bands and the grayscale image captured simultaneously. The targets are surrounded by "homogenous" grass. On the top and bottom of the image experimental plots can partially be seen. These were separated by applying herbicides resulting in paths of 
dry grass. Some of these can be identified in the NDVI image. Besides, the reference panels and the ground control points are clearly visible. When taking a close look at the top right corner of the hyperspectral images the already mentioned erroneous pattern can be observed.

\section{DISCUSSION AND CONCLUSION}

The goal of this study was to develop a calibration methodology for hyperspectral full-frame sensors. Additionally, we wanted to evaluate important characteristics (like the dark current and the real dynamic range) and consider the feasibility of the UHD 185 full-frame hyperspectral imager for vegetation studies.

We found a linear behavior of the UHD 185 for a wide range of illumination intensities. At a DN higher than 3600 (out of 4096) the quantum efficiency decreased significantly. The results also show a nonlinear behavior at low DN values. Since the latter effect is a not known for CCDs it might result from the very low light intensities and eventually non-homogeneous illumination conditions inside the integration sphere. For the specific case of vegetation studies, were the reflectance of the visible part of the spectrum is significantly lower than the reflectance in the near infrared a rDR of about 3600 still leaves a fair amount of dynamic range for the visible region. Additionally, low dark current only has a negligible effect. With these results the sensor shows a good signal to noise ratio. However, the dark current should be evaluated under in-field conditions were the sensor might heat up more due to the sun's radiation and higher air temperature. For a more precise dark current correction a temperature sensor would be beneficial.

During the assessment of the vignetting effect an overlying pattern with a presumable origin in the sensor's optical system was observed. Together both effects resulted in a DN difference of up to 90 percent between the brightest and darkest pixel. Despite these effects are relatively decreasing with light intensity they significantly reduce the rDR range of the sensor towards the edges of the image. Latter needs to be further evaluated. To improve the overall signal to noise ratio in remote sensing of vegetation it might be considered to apply a filter which blocks some of the NIR radiation. However, this filter would bring the disadvantage of longer integration times.

The LUT approach yielded good results in compensating the inhomogeneous illumination of the CCD. Different coefficient for different pixels highlight the need for band specific calibrations. The derived LUT coefficients provided stable results for different illumination conditions. The slight inhomogeneity with increasing illumination might be attributed to slight inhomogeneity inside the integration sphere when not all light sources are used. This is supported by the spatial trend across the flat-fielded images with changed illumination (Figure 6 , right).

When applying the LUT to an in-field measurement of a Zenith white panel a residual undulated pattern appears. Between the trough and the crest the DN values differentiate by up to 15 percent. Currently, the origin of this effect is still unknown. Despite it is not obvious in real in-field scenes (Figure 8) this error needs further investigation.

In this paper we introduced a modified pre-flight calibration were a flat-fielded measurement was used to translate the measured DN values to reflectance values. This approach yielded very good results leading to an offset smaller than 3 percent for 110 of the 125 bands when compared with measurements of an ASD FieldSpec 3. Above $886 \mathrm{~nm}$ (band 110) the offset rapidly increases which might result from the decreasing sensitivity of $\mathrm{Si}$ CCDs and the general lower light availability in this spectral region. Compared with a standard pre-flight calibration, where the raw image of a reference target is used, the introduced new approach is less prone to disturbances by the sensor-calibration target geometry. In Figure 6 (right) a subordinated trend of higher illumination across the calibration panel becomes obvious after the calibration. With the standard calibration approach, this would have been introduced to all measurements transformed with this reference.

One of the biggest benefits of UAV remote sensing is the ability to gather optical measurements below the clouds. However, due to changing illumination conditions this requires constant measurements compensation of the change in incoming radiation. Even under cloudless conditions monitoring the illumination conditions becomes increasingly important with increasing flight duration and extended spatial coverage of UAVs. Thus, lightweight irradiance sensors should be developed and deployed with mobile hyperspectral sensor systems.

Overall, the presented results align with the experiences in the field which show that the UHD 185 is suitable for hyperspectral remote sensing of agricultural crops. The short integration time reduces image blur and allow adaption to less illuminated conditions due to cloud cover. Besides of the hyperspectral data three dimensional crop surface models may be derived since the sensor captures full image frames. These serve as a complementary information for crop growth monitoring (Bendig et al., 2013).

However, as mentioned in this paper, some uncertainties still remain. These will be targeted in future work. Additionally, the automated processing chain developed for the processing of hyperspectral data of full-frame sensors will be optimized.

\section{ACKNOWLEDGMENTS}

The authors would like to thank Andreas Burkart and Uwe Rascher from the IBG-2 at the research center Jülich, Germany, for the access to and their support with the measurements at the integration sphere. Additionally, the authors would like to thank Rene Michels and Rainer Graser from the Cubert GmbH for the open and constructive discussions and their support. Also, the authors acknowledge the funding of the CROP.SENSe.net project in the context of the Ziel 2-Programms NRW 2007-2013 "Regionale Wettbewerbsfähigkeit und Beschäftigung" by the Ministry for Innovation, Science and Research (MIWF) of the state North Rhine Westphalia (NRW) and European Union Funds for regional development (EFRE) (005-1103-0018).

\section{LITERATURE}

Aasen, H., Gnyp, M.L., Miao, Y., Bareth, G., 2014. Automated Hyperspectral Vegetation Index Retrieval from Multiple Correlation Matrices with HyperCor. Photogramm. Eng. Remote Sens. 80, 785-795.

Atzberger, C., 2013. Advances in Remote Sensing of Agriculture: Context Description, Existing Operational Monitoring Systems and Major Information Needs. Remote Sens. 5, 949-981.

Bareth, G., Aasen, H., Bendig, J., Gnyp, M.L., Bolten, A., Jung, A., René Michels, J., Souk-Kamäki, U., in review. Lowweight and UAV-based hyperspectral full-frame cameras for monitoring crops: spectral comparison with portable spectroradiometer measurements.

Bendig, J., Bolten, A., Bareth, G., 2013. UAV-based Imaging for Multi-Temporal, very high Resolution Crop Surface Models to monitor Crop Growth Variability Monitoring des Pflanzenwachstums mit Hilfe multitemporaler und 
hoch auflösender Oberflächenmodelle von

Getreidebeständen auf Basis von Bilder. Photogramm. Fernerkundung - Geoinf. 2013, 551-562.

Berni, J.A.J., Member, S., Zarco-tejada, P.J., Suárez, L., Fereres, E., 2009. Thermal and Narrowband Multispectral Remote Sensing for Vegetation Monitoring From an Unmanned Aerial Vehicle 1-17.

Burger, J., Geladi, P., 2006. Hyperspectral NIR image regression part I : Calibration and correction 355-363.

Burkart, A., Cogliati, S., Schickling, A., Rascher, U., 2014. A Novel UAV-Based Ultra-Light Weight Spectrometer for Field Spectroscopy. IEEE Sens. J. 14, 62-67.

Delalieux, S., van Aardt, J., Keulemans, W., Schrevens, E., Coppin, P., 2007. Detection of biotic stress (Venturia inaequalis) in apple trees using hyperspectral data: Nonparametric statistical approaches and physiological implications. Eur. J. Agron. 27, 130-143.

Erdle, K., Mistele, B., Schmidhalter, U., 2011. Comparison of active and passive spectral sensors in discriminating biomass parameters and nitrogen status in wheat cultivars. F. Crop. Res. 124, 74-84.

Foley, J. a, Defries, R., Asner, G.P., Barford, C., Bonan, G., Carpenter, S.R., Chapin, F.S., Coe, M.T., Daily, G.C., Gibbs, H.K., Helkowski, J.H., Holloway, T., Howard, E. a, Kucharik, C.J., Monfreda, C., Patz, J. a, Prentice, I.C., Ramankutty, N., Snyder, P.K., 2005. Global consequences of land use. Science 309, 570-4.

Foley, J. a, Ramankutty, N., Brauman, K. a, Cassidy, E.S., Gerber, J.S., Johnston, M., Mueller, N.D., O'Connell, C., Ray, D.K., West, P.C., Balzer, C., Bennett, E.M., Carpenter, S.R., Hill, J., Monfreda, C., Polasky, S., Rockström, J., Sheehan, J., Siebert, S., Tilman, D., Zaks, D.P.M., 2011. Solutions for a cultivated planet. Nature 478, 337-42.

Gnyp, M.L., Yu, K., Aasen, H., Yao, Y., Huang, S., Miao, Y., Bareth, G., 2013. Analysis of Crop Reflectance for Estimating Biomass in Rice Canopies at Different Phenological Stages Reflexionsanalyse zur Abschätzung der Biomasse von Reis in unterschiedlichen phänologischen Stadien. Photogramm. - Fernerkundung Geoinf. 2013, 351-365.

Goldman, D.B., 2010. Vignette and exposure calibration and compensation. IEEE Trans. Pattern Anal. Mach. Intell. $32,2276-88$.

Green, R., Eastwood, M., 1998. Imaging spectroscopy and the airborne visible/infrared imaging spectrometer (AVIRIS). Remote Sens. ... 4257.

Hansen, P.M., Schjoerring, J.K., 2003. Reflectance measurement of canopy biomass and nitrogen status in wheat crops using normalized difference vegetation indices and partial least squares regression $86,542-553$.
Honkavaara, E., Saari, H., Kaivosoja, J., Pölönen, I., Hakala, T., Litkey, P., Mäkynen, J., Pesonen, L., 2013. Processing and Assessment of Spectrometric, Stereoscopic Imagery Collected Using a Lightweight UAV Spectral Camera for Precision Agriculture. Remote Sens. 5, 5006-5039.

Hruska, R., Mitchell, J., Anderson, M., Glenn, N.F., 2012. Radiometric and Geometric Analysis of Hyperspectral Imagery Acquired from an Unmanned Aerial Vehicle. Remote Sens. 4, 2736-2752.

Kim, S.J., Pollefeys, M., 2008. Robust radiometric calibration and vignetting correction. IEEE Trans. Pattern Anal. Mach. Intell. 30, 562-76.

Li, F., Miao, Y., Hennig, S.D., Gnyp, M.L., Chen, X., Jia, L., Bareth, G., 2010. Evaluating hyperspectral vegetation indices for estimating nitrogen concentration of winter wheat at different growth stages. Precis. Agric. 11, 335357.

Lucieer, A., Malenovský, Z., Veness, T., Wallace, L., 2014. HyperUAS-Imaging Spectroscopy from a Multirotor Unmanned Aircraft System. J. F. Robot. 31, 571-590.

Mahlein, A., Rumpf, T., Welke, P., Dehne, H., Plümer, L., Steiner, U., Oerke, E., 2013. Remote Sensing of Environment Development of spectral indices for detecting and identifying plant diseases. Remote Sens. Environ. 128, 21-30.

Mansouri, a., Marzani, F.S., Gouton, P., 2005. Development of a Protocol for CCD Calibration: Application to a Multispectral Imaging System. Int. J. Robot. Autom. 20.

Milton, E.J., Schaepman, M.E., Anderson, K., Kneubühler, M., Fox, N., 2009. Progress in field spectroscopy. Remote Sens. Environ. 113, S92-S109.

Mulla, D.J., 2013. Twenty five years of remote sensing in precision agriculture: Key advances and remaining knowledge gaps. Biosyst. Eng. 114, 358-371.

Pearlman, J.S., Barry, P.S., Segal, C.C., Shepanski, J., Beiso, D., Carman, S.L., 2003. Hyperion, a space-based imaging spectrometer. IEEE Trans. Geosci. Remote Sens. 41, $1160-1173$.

Ray, D.K., Mueller, N.D., West, P.C., Foley, J. a, 2013. Yield Trends Are Insufficient to Double Global Crop Production by 2050. PLoS One 8, e66428.

Stagakis, S., González-Dugo, V., Cid, P., Guillén-Climent, M.L., Zarco-Tejada, P.J., 2012. Monitoring water stress and fruit quality in an orange orchard under regulated deficit irrigation using narrow-band structural and physiological remote sensing indices. ISPRS J. Photogramm. Remote Sens. 71, 47-61.

Suomalainen, J., Anders, N., Iqbal, S., Roerink, G., Franke, J., Bartholomeus, H., Becker, R., Kooistra, L., Sensing, R., Physics, S., Group, L.M., 2014. remote sensing. 
The International Archives of the Photogrammetry, Remote Sensing and Spatial Information Sciences, Volume XL-7, 2014 ISPRS Technical Commission VII Symposium, 29 September - 2 October 2014, Istanbul, Turkey

Yu, K., Leufen, G., Hunsche, M., Noga, G., Chen, X., Bareth, G., 2013a. Investigation of Leaf Diseases and Estimation of Chlorophyll Concentration in Seven Barley Varieties Using Fluorescence and Hyperspectral Indices. Remote Sens. 6, 64-86.

Yu, K., Li, F., Gnyp, M.L., Miao, Y., Bareth, G., Chen, X., 2013b. Remotely detecting canopy nitrogen concentration and uptake of paddy rice in the Northeast China Plain. ISPRS J. Photogramm. Remote Sens. 78, 102-115.

Yu, W., 2004. Practical anti-vignetting methods for digital cameras. IEEE Trans. Consum. Electron. 50, 975-983. 